\title{
Cerebellar cryptococcoma in a non-HIV patient
}

\author{
Fabiano Reis ${ }^{1}$, Ricardo Schwingel ${ }^{1}$, Thiago Ferreira Souza', \\ Luciano de Souza Queiroz ${ }^{2}$, Verônica de Araújo Zanardi'
}

A 26-year-old female, immunocompetent, with dysmetria and dysarthria. Diagnostic imaging (Fig 1) demonstrated a cerebellar mass, with a pattern which may be associated to a tumor, although multiple hypointense centers surrounded by ring enhancement after gadolinium may suggest the possibility of many types of infectious lesions, including cryptococcoma.

Histological analysis (Fig 2) confirmed the diagnosis of Cryptococcus neoformans.

Central nervous system cryptococcosis is rare in immunocompetent individuals and is considered an opportunistic pathogen in AIDS patients ${ }^{1-3}$. In immunocom- petent individuals, cryptococcoma is the most common form of cerebral involvement ${ }^{1,2}$.

This patient had a good outcome after surgery and anti-fungal therapy.

\section{REFERENCES}

1. Kesler R, Maertens P. Pontine cryptococcoma in a nonimmunocompromised individual: MRI characteristics. J Neuroimaging 1999;9:118-121.

2. Li Q, You C, Liu Q, Liu Y. Central nervous system cryptococcoma in immunocompetent patients: a short review illustrated by a new case. Acta Neurochir 2010;152:129-136.

3. Rocha AJ, Maia Junior ACM, Ferreira NPDF, Amaral LLF. Granulomatous diseases of the central nervous system. Top Magn Reson Imaging 2005; 16:155-187.
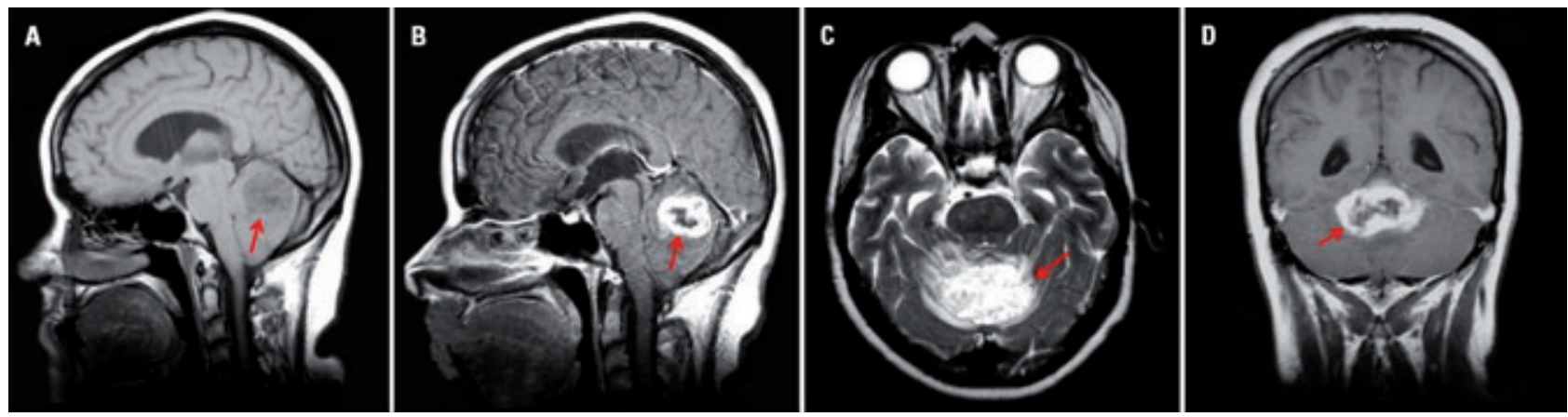

Fig 1. [A] Sagittal T1 showing a cerebellar hypointense lesion. [B]Sagittal T1 after gadolinium showing a vermian mass with multiple hypointense centers with ring enhancement. [C] Axial T2 reveals a hyperintense lesion, with edema surrounding the mass. [D] Coronal T1, after gadolinium showing the cerebellar lesion with ring enhancement.
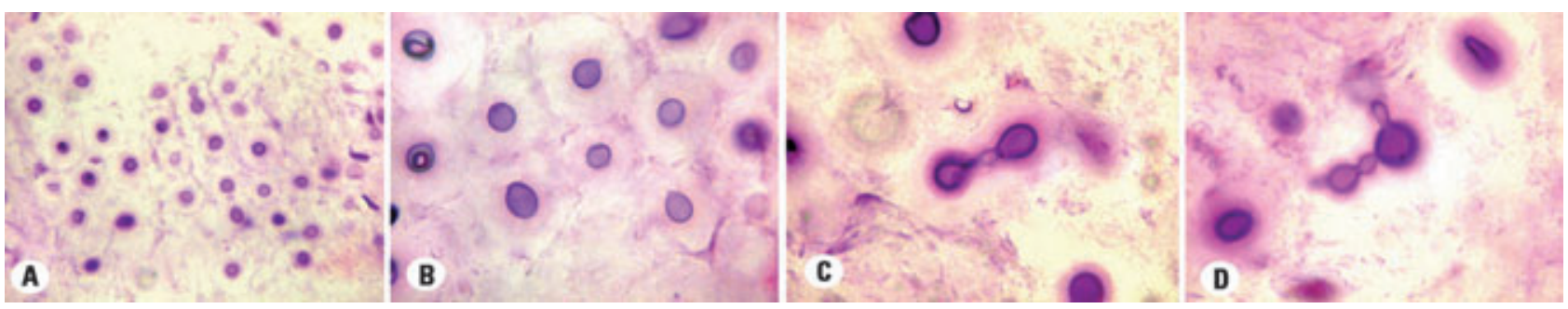

Fig 2. All pictures: smear preparation stained with HE. $[A] \times 400$. [B], [C] and [D] $\times 1000$. [A] Abundant fungi in smear from cerebellar lesion. [B] Yeast forms surrounded by thick mucinous capsule which keeps fungus cells apart. [C] and [D] Reproduction occurs by single or multiple budding.

\section{CRIPTOCOCOMA CEREBELAR EM PACIENTE HIV NEGATIVO}

'Department of Radiology of the Clinics Hospital of the State University of Campinas, Faculty of Medical Sciences, Campinas SP, Brazil; ${ }^{2}$ Department of Pathology of the Clinics Hospital of the State University of Campinas, Faculty of Medical Sciences, Campinas SP, Brazil.

Correspondence: Fabiano Reis - Faculdade de Ciências Médicas / Universidade Estadual de Campinas / Departamento de Radiologia - Rua Tessália Vieira de Camargo 126 - Caixa Postal: 6111 / Cidade Universitária Zeferino Vaz - 13083-887 Campinas SP - Brasil. E-mail: fabianoreis2@gmail.com

Support: Ricardo Schwingel received financial support by FAPESP.

Received 3 December 2010. Received in final form 2 May 2011. Accepted 9 May 2011. 